\title{
Harm: albumin neither increases nor decreases mortality in critically ill patients
}

\section{Article appraised}

Wilkes MM, Navickis RJ. Patient survival after human albumin administration. A meta-analysis of randomized, controlled trials. Ann Intern Med 2001; 135: 149-64.

\section{Structured abstract}

Question: In critically ill patients, is there a difference in mortality between patients who receive human albumin compared to patients who do not receive human albumin?

Data sources: Studies were identified by computerized searches (MEDLINE, EMBASE, Cochrane Controlled Trials Register, Cochrane Medical Editors Trial Amnesty), internet searches, hand searches of selected medical journals (January 1990 to November 2000 issues of BMJ, JAMA, Lancet, and $N$ Engl J $M e d)$, citation review, and contact of authors. No language restrictions were applied.

Study selection: Studies were selected if they were randomized controlled trials; compared albumin therapy with: 1) no therapy, 2) crystalloid therapy, or 3) a lower dose of albumin; and contained mortality data.

Data extraction: Data were extracted in duplicate on trial design, patient characteristics, and mortality. Unpublished mortality data were sought from trial investigators.

Main results: Fifty-five trials with a total of 3,504 patients met the inclusion criteria. Forty-two of 55 trials reported at least one death. Albumin therapy did not change mortality rates in patients undergoing surgery, patients with trauma, burns, hypoalbuminemia, ascites, or other critical conditions, or high-risk neonates (Table). Sensitivity analyses of blinding, mortality as an a priori outcome, and crossover did not show any statistical differences in mortality. There was statistically significant $(P=0.03)$ publication bias with small-sized trials (each with $<100$ patients) favouring no albumin.

Conclusions: Albumin therapy neither increases nor decreases mortality in critically ill patients.

Funding: The Plasma Protein Therapeutics Association and the American Red Cross.

Correspondence: Mahlon M. Wilkes, PhD, Hygeia Associates, 17988 Brewer Road, Grass Valley, CA 95949, USA. Email: mwilkes@hygeiaassociates.com

TABLE Effect of albumin therapy on mortality

\begin{tabular}{|c|c|c|c|c|}
\hline Trial category & $\begin{array}{l}\text { Events } \\
\text { Albumin }\end{array}$ & Control* $^{*}$ & $\begin{array}{l}\text { Relative riskt } \\
(95 \% \mathrm{CI})\end{array}$ & P value \\
\hline All patients & $273 / 1456$ & $252 / 1502$ & $1.11(0.95-1.28)$ & Not significant \\
\hline Surgery/trauma & $87 / 650$ & $78 / 689$ & $1.12(0.85-1.46)$ & Not significant \\
\hline Burns & $24 / 95$ & $15 / 102$ & $1.76(0.97-3.17)$ & Not significant \\
\hline Hypoalbuminemia & $27 / 184$ & $17 / 173$ & $1.59(0.91-2.78)$ & Not significant \\
\hline Ascites & $49 / 185$ & $54 / 188$ & $0.93(0.67-1.28)$ & Not significant \\
\hline Other $\ddagger$ & $50 / 190$ & $58 / 198$ & $0.91(0.67-1.22)$ & Not significant \\
\hline High-risk neonates & $36 / 152$ & $30 / 152$ & $1.19(0.78-1.81)$ & Not significant \\
\hline
\end{tabular}

*Control group includes subjects who received no therapy, crystalloid therapy, or lower doses of albumin. †Relative risk less than 1 favours the albumin group (i.e., albumin is beneficial); relative risk greater than 1 favours the control group (i.e., albumin is harmful). $\ddagger$ Patients had acute respiratory distress syndrome, septic or hypovolemic shock, or acute ischemic stroke. 


\section{Commentary by G. Bryson}

Wilkes and Navickis present a systematic review evaluating the influence of albumin administration on mortality. This is a well-conducted review by the standards cited in the Users' Guides to the Medical Literature. ${ }^{1}$ The review presents a focused clinical question, a rigorous search of the literature, appropriately selected articles, duplicate evaluation, and a variety of sensitivity analyses based upon study quality. No statistically significant increase in mortality was found when albumin was used in surgery/trauma (relative risk (RR) $1.12,95 \%$ confidence interval (CI) 0.85 to 1.46 ), burns (RR 1.76, 95\% CI 0.97 to 3.17 ), or when all indications were pooled (RR 1.11, 95\% CI 0.95 to 1.28). Should the results of this systematic review "allay concerns regarding the safety of albumin" as the authors suggest?

One should always remain cautious when interpreting the results of a systematic review, even a good one.

"The combining of heterogeneous material is a commonly accepted threat to the validity of metaanalysis". 2 More simply stated, you can't combine apples and oranges. Statistical tests for heterogeneity in this review were negative but substantial clinical heterogeneity remains. The surgery/trauma subgroup included studies of albumin for acute normovolemic hemodilution, priming of cardiopulmonary bypass circuits, preservation of orthotopic liver transplants, and direct management of colloid osmotic pressure. A clinician would be unlikely to consult these sources when trying to decide what fluid to give the hypotensive patient in the operating room.

It is also easy to be drawn in by the simplicity of a single number to summarize the results of all this research. The 95\% CI surrounding the estimates of albumin's effect are not statistically significant, leading one to conclude that albumin is harmless. One should be more critical. Most subgroups in this study demonstrated an excess of mortality in the group treated with albumin. The $95 \%$ CI of all analyses include the possibility of clinically relevant harm and in no case did the use of albumin create statistically or clinically significant reductions in mortality. The statistical absence of harm does not prove safety.

Lastly, it is important to place the results of this systematic review in the context of previous research. The Cochrane Injuries Group Albumin Reviewers found that albumin increased the risk of mortality (RR $1.52,95 \%$ CI 1.17 to 1.99 ) and concluded that "there is no evidence that albumin administration reduces the risk of death in critically ill patients...". ${ }^{3}$ Similarly Choi and colleagues found a trend to lower mortality with crystalloid resuscitation (RR $0.86,95 \%$ CI 0.63 to 1.17) and stated that "there is no apparent difference in pulmonary edema, mortality, or length of stay between isotonic crystalloid and colloid". ${ }^{4}$

So should clinicians feel safe in choosing albumin? Despite differences in methods and interpretation, all systematic reviews are unanimous in showing no clinical benefit to albumin therapy. Without clear benefit and with unresolved concerns regarding the transmission of new variant Creutzfeld Jacob disease in human blood products, ${ }^{5}$ a compelling argument for the use of albumin is difficult to make.

Gregory L. Bryson MD FRCPC MSc

Ottawa, Ontario

\section{References}

1 Oxman AD, Cook DJ, Guyatt GH, for the EvidenceBased Medicine Working Group. Users' guides to the medical literature. VI. How to use an overview. JAMA 1994; 272: 1367-71.

2 Chalmers TC. Problems induced by meta-analyses. Stat Med 1991; 10: 971-9; discussion 979-80.

3 The Albumin Reviewers. Human albumin solution for resuscitation and volume expansion in critically ill patients (Cochrane review). In: The Cochrane Library, Issue 1, 2002. Oxford: Update Software. Document CD001208.

4 Choi PTL, Yip G, Quinonez LG, Cook DJ. Crystalloids vs. colloids in fluid resuscitation: a systematic review. Crit Care Med 1999; 27: 200-10.

5 Department of Health. Further precautionary measures on blood products announced. Available from: http://www.doh.gov.uk/cjd/press/measures.htm

\section{Commentary by P. Choi}

The long-standing debate about the use of crystalloids or albumin in seriously ill patients is one that has been brought to the public's attention with the increased awareness of risks of human blood products and the 1998 publication of a systematic review on this topic. ${ }^{1}$ Wilkes and Navickis present another synthesis of the evidence on this subject. Their review has many strengths: comprehensive search strategy, explicit selection criteria, duplication selection and abstraction of eligible randomized controlled trials (RCTs), evaluation of methodological quality, clear data analysis, and transparent reporting of their study based on the QUOROM (Quality of Reporting of Meta-Analyses) format. ${ }^{2}$

This systematic review raises several important issues. First, research findings must be interpreted with caution. Wilkes and Navickis report non-statistically significant differences in mortality for all patients and for various subgroups. They conclude that " $[t]$ his finding 
supports the safety of albumin". Closer scrutiny of their results suggests that, at best, albumin neither decreased nor increased mortality and that, at worst, the results failed to show a statistically significant increase in mortality due to inadequate patient numbers from current studies. For all but the subgroups for ascites and "other", the point estimates of relative risk suggested harm. The $95 \%$ confidence interval estimates the range within which the true effect of albumin lies 19 out of 20 times: the interval is consistent with benefit and harm in all groups. In the worst case scenario, compared to crystalloids, albumin may result in a relative overall increase in mortality of $28 \%$ in all patients and an increase of $46 \%$ in surgical or trauma patients. It is still too soon to conclude that albumin is safe.

Second, although systematic reviews can inform clinical practice, "garbage in" will result in "garbage out". Of the 55 included RCTs, six were blinded and 21 had adequate allocation concealment. The median sample size was 58 patients. Demonstration of efficacy requires plausible biological mechanism, improvement in clinically relevant outcomes (or reduction of clinical morbidity and mortality) in a population and setting that is reflective of current clinical practice, and an adequate sample size to detect the effect. Wilkes and Navickis's review demonstrates the heterogeneity in interventions, study populations, clinical settings, and end points between studies and the lack of statistical power of these studies to demonstrate a difference. Clearly, we need large RCTs to answer our questions on fluid choice.

Third, this review identifies, by their absence, populations in which fluid choice may be important but randomized studies have not yet been conducted. For example, patients with head trauma, parturients with pre-eclampsia or eclampsia, and pediatric patients would merit further research. So far, very few RCTs on fluid choice have been conducted in these populations; none have examined mortality as an outcome.

In summary, this review provides a synthesis of the current evidence on patient survival after albumin administration and indicates that further research is needed before we can draw any conclusions on the safety of this human blood product.

Peter T. Choi MD FRCPC MSc

Hamilton, Ontario

\section{References}

1 Cochrane Injuries Group Albumin Reviewers. Human albumin administration in critically ill patients: systematic review of randomised controlled trials. BMJ 1998; 317: 235-40.
2 Moher D, Cook DJ, Eastwood S, Olkin I, Rennie D, Stroup DF, for the QUOROM Group. Improving the quality of reports of meta-analyses of randomised controlled trials: the QUOROM statement. Quality of reporting of meta-analyses. Lancet 1999; 354: 1896-900. 